\title{
Facile Fabrication of Polyimide-alumina Composite Coatings by Liquid Flame Spray
}

\author{
Jingzhong Zhou ${ }^{1}$, Kuoteng Sun ${ }^{1}$, Songqiang Huang ${ }^{1}$, Xuemin He ${ }^{1}$, Weichen Cai ${ }^{1}$, \\ Yuantao Zhao ${ }^{2, *}$ and Wenge $\mathrm{Li}^{2, *}$ \\ 1 Liuzhou Bureau of EHV Transmission Company, China Southern Power Grid Co., Ltd. \\ Liuzhou 545006, China; zjzking@163.com (J.Z.); sunkuoteng@163.com (K.S.); foobarx@163.com (S.H.); \\ hexuemin012@163.com (X.H.); caiweichen93@163.com (W.C.) \\ 2 Merchant Marine College, Shanghai Maritime University, Shanghai 201306, China \\ * Correspondence: zhaoyt@shmtu.edu.cn (Y.Z.); wgli@shmtu.edu.cn (W.L.)
}

Received: 20 June 2020; Accepted: 2 September 2020; Published: 4 September 2020

\begin{abstract}
Polyimide is type of heterocyclic polymer compound characterized by an imide ring structure. The aromatic ring structure in polyimide facilitates good electrical properties, mechanical properties, thermal properties and radiation resistance, etc. It is widely used in the aerospace field, in electronic components and in the semiconductor industry. Traditional methods for the preparation of polyimide films have different limitations. Here, a new one-step method was established to prepare polyimide coatings using liquid flame spray. The effect of different mass fraction on the surface and cross section structure characteristics of polyimide coatings was studied. The formation process of the polyimide-alumina composite coatings was analyzed using single particles and coatings with different amount of alumina. The composite coating shows a flat and dense structure and excellent dielectric properties. The results shed light on construction of polymer-inorganic composite layers for widespread applications.
\end{abstract}

Keywords: polyimide; composite; coating; liquid flame spray; dielectric property

\section{Introduction}

Polyimide material has good comprehensive performance and is currently the best temperature-resistant polymer material in industrial applications. Polyimide also has excellent electrical and mechanical properties and is widely used as an electrical insulation material $[1,2]$. It generally begins to decompose at about $500{ }^{\circ} \mathrm{C}$ and can also withstand extremely low temperatures. The dielectric constant and tensile strength do not change significantly with temperature and frequency. As an engineering plastic, the elastic modulus of polyimide fiber can reach $200 \mathrm{GPa}$, showing a low coefficient of thermal expansion and a high radiation resistance [3].

Through the modification of inorganic particles and the development of inorganic particles with special functions, polyimide/inorganic hybrid composite coatings were studied to improve the structure and performance of polyimide. A large amount of research is currently focused on inorganic particles such as carbon nanotubes, graphene, boron nitride, $\mathrm{WO}_{3}, \mathrm{Al}_{2} \mathrm{O}_{3}, \mathrm{SiO}_{2}$, etc. [4-11]. Recent studies focused on the preparation of polyimide microspheres [12], which have a larger specific surface area, better dispersibility in solvents, and improved processing performance and temperature resistance, giving polyimide materials a wider range of applications.

Polyimide is generally prepared by a two-step method, with the precursor solution of polyimide being prepared first and then the formation of polyimide by imidization reaction $[13,14]$. The traditional methods for preparing polyimide film mainly include the sol-gel method, the spin coating method, the vapor deposition method, the one-step method, the two-step method, etc., and the thermal spray 
method is rarely used to prepare polyimide coating [15]. This article aims to study the preparation of polyimide coatings by thermal spray and find suitable parameters to obtain polyimide and composite coatings with good structure and performance for application in fields such as electrical insulation.

\section{Materials and Methods}

Polyimide precursor solution was prepared using monomer pyromellitic dianhydride (PMDA, 80,112,127 CP, Sinopharm Chemical Reagent Co. Ltd., Beijing, China), 4,4'-oxydianiline (ODA, 30049926CP, Sinopharm Chemical Reagent Co. Ltd., Beijing, China) and dimethylformamide (DMF) solvent. Briefly, $2.0024 \mathrm{~g}$ ODA was dissolved in $40 \mathrm{~mL}$ DMF with stirring for $5 \mathrm{~min}$. Then $2.1812 \mathrm{~g}$ PMDA was slowly added into the solution with vigorous stirring and continued stirring for $8-10 \mathrm{~h}$ to obtain the polyimide precursor polyamic acid (PAA) solution. This solution corresponds to a mass fraction of $10 \%$ and was diluted to the specified concentration by DMF before coating deposition. The coatings were deposited by flame spray (CDS 8000, Castolin, Germany) on 316L stainless steel plates with the dimension of $25 \mathrm{~mm} \times 20 \mathrm{~mm} \times 1.5 \mathrm{~mm}$. Before use, the stainless-steel plates were washed with aceton, ethanol and water in order and then sand blasted after air drying. The prepared solution was injected into the flame using a spray gun powered by compressed air. Oxygen and acetylene were used as the combustion-supporting and fuel gas withpressures of 0.5 and $0.1 \mathrm{MPa}$, respectively. Nanoalumina powders of 1000 mesh were obtained from Guangzhou Kingsky Material, Guangzhou, China.

Morphology of the splats and the coatings was characterized by field emission scanning electron microscope (FESEM, FEI Quanta FEG250, Amsterdam, The Netherlands).

\section{Results and Discussion}

The polyimide precursor solution PAA prepared here has a mass fraction of $10 \%$. The viscosity of the $10 \%$ PAA precursor solution is slightly larger, making it difficult to atomize. Dilution of the PAA solution does not change the molecular weight of the PAA, and will not subsequently affect the molecular weight of the imidized polyimide; while dilution of the PAA solution can reduce the viscosity of the solution, which is beneficial to the solution atomization. However, too much solvent content in the atomized particles and fewer components of PAA will reduce thermal imidization of the particles before reaching the matrix and lead to the formation of large bubbles when spraying. Therefore, the precursor solution was diluted to a mass fraction of $5 \%$ and $8 \%$ for the spraying test.

It can be seen from Figure 1 that the surface of both coatings contains pores. The diameters of the pores are about $50 \mu \mathrm{m}$ and $30 \mu \mathrm{m}$, in Figure 1a,b, respectively. And the content of mesopores in Figure $1 b$ is significantly less than that in Figure $1 a$. This indicates that a higher mass fraction in particles shows better solvent evaporation by heating and increases the concentration of PAA, which is beneficial to the thermal imidization process to generate polyimide. The amic acid reaching the substrate continues to be imidized, and the generated gas escapes from the post-sprayed coating and forms pores in the coating.

The cross-sectional structure of the coating was then examined. As shown in Figure 2, the content of pores in the coating from 5\% PAA is significantly more than that in $8 \%$ PAA. Furthermore, most of the pores in the coating from 5\% PAA exist in the form of through-holes, while most of the pores from $8 \%$ PAA are closed pores. This suggests that there is more PAA in a single particle from the precursor solution with higher mass fraction, which leads to better cross-linking degree and mechanical properties. Therefore, $8 \%$ PAA was selected for the further fabrication of composite coatings.

As a typical inorganic powder, $\mathrm{Al}_{2} \mathrm{O}_{3}$ is widely used in the production of composite PI films for modification of structure and performance. Therefore, we chose it as an example to verify our technique for the one-step fabrication of PI composite coatings. In this process, $10 \%$ content of alumina nano powders were added into $8 \%$ PAA solutions, and its effect on the single particle nucleation process in the spraying process of PAA was first studied. Figure 3a shows the spread of a single atomized droplet on the substrate, and Figure $3 \mathrm{~b}$ shows a partial enlarged view of a single droplet. It is clearly 
shown that polyimide formed in multiple places within a single droplet, indicating that this exhibits a heterogeneous nucleation process during the spraying process of PAA with alumina powders.

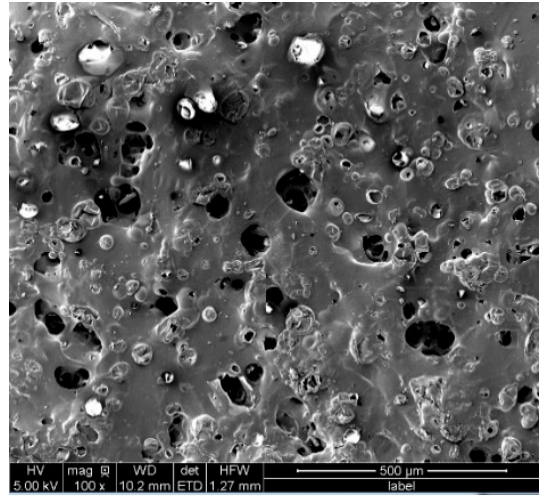

(a)

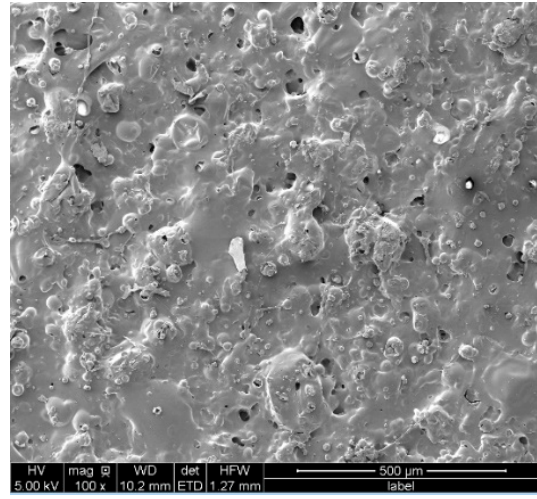

(b)

Figure 1. SEM images of coatings fabricated using 5\% PAA (a) and 8\% PAA (b).

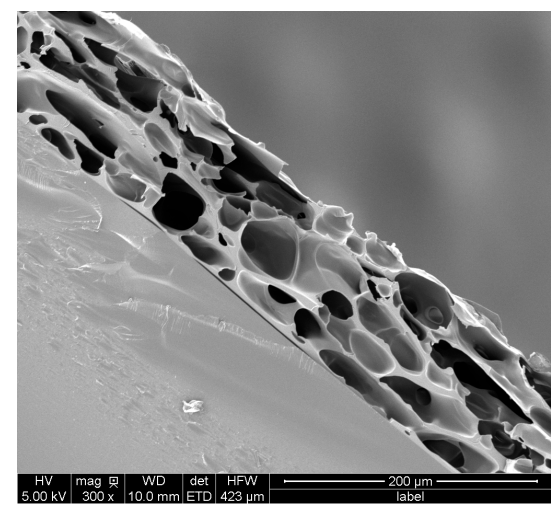

(a)

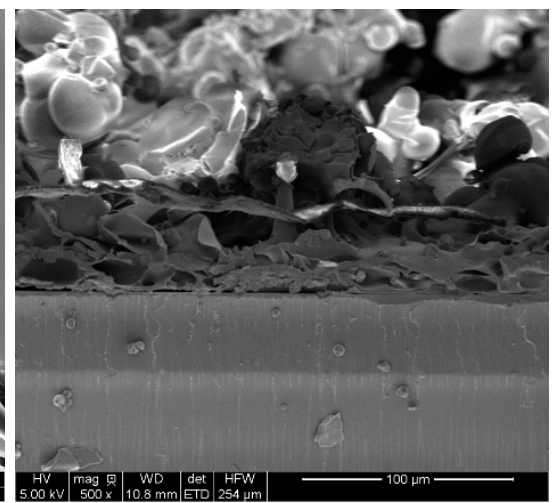

(b)

Figure 2. Cross-sectional structure of coatings fabricated using 5\% PAA (a) and 8\% PAA (b).

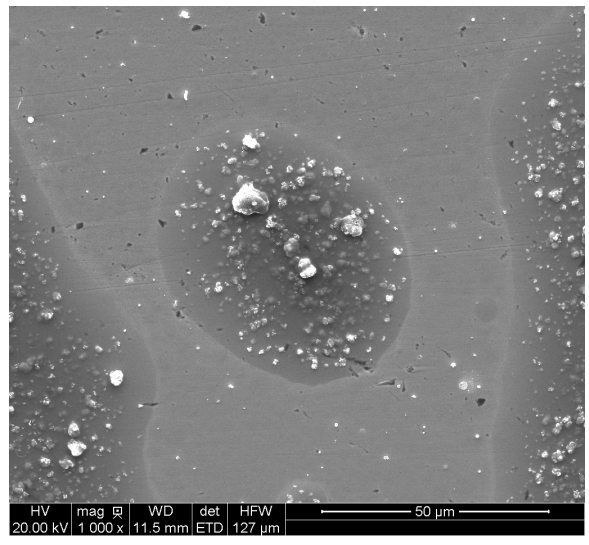

(a)

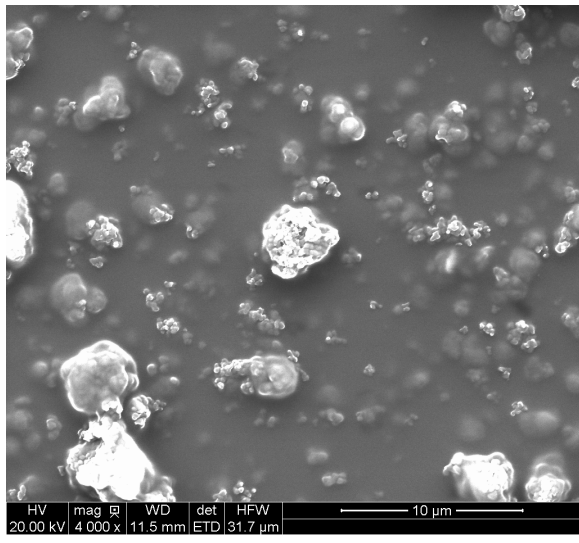

(b)

Figure 3. (a) A single polyimide droplet with $10 \%$ alumina and (b) a partial enlarged view of the droplet.

Subsequently, different contents of nano-alumina were dispersed in the PAA solution, and used to produce composite coatings by flame spray with the same spraying parameters. The surface and cross-sectional morphology of these coatings were examined. As shown in Figure 4, the 30\% aluminum oxide composite coating has a greater doping amount of inorganic particles and more nucleation of atomized particles, resulting in increased unevenness of the coating surface and the formation 
of protrusions. The surface characteristics of the inorganic hybrid composite polyimide coating are basically flat compared with the pure polyimide coating with no obvious holes. The cross-sectional structural diagrams of polyimide inorganic hybrid alumina composite coatings with different contents was shown in Figure 5. The coating with 30\% aluminum oxide is denser and has less pore content than $10 \%$ and $20 \%$ coatings. Most of the pores in these coatings are closed pores. Coating layer cross-linking is also better, and the resulting coating is relatively complete. This suggests that the addition of the heterogeneous nucleating agent enables the single particles to form multiple polyimide nuclei in the flame and gradually grow, leading to high degree of imidization and low rate of residual solvent. The single-layer coating will not generate too much gas during the subsequent spray heating process, which effectively reduces the content of pores and the number of through-holes.

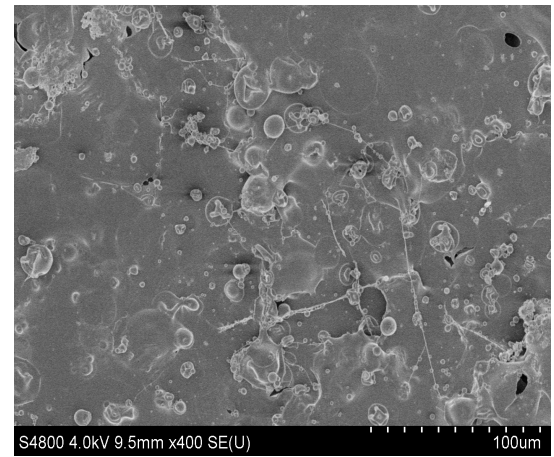

(a)

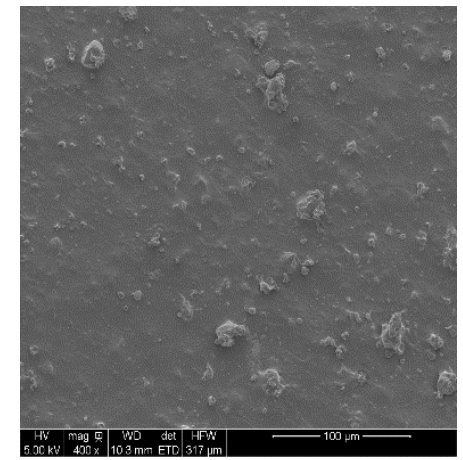

(b)

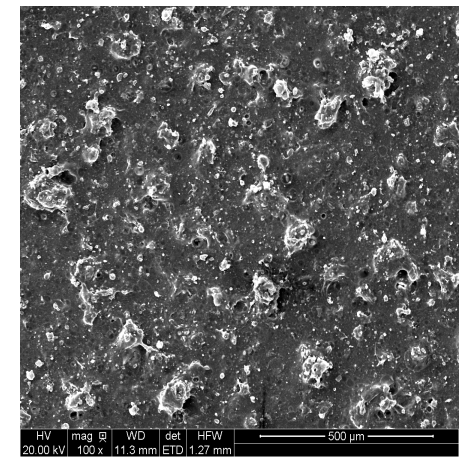

(c)

Figure 4. Surface structure of polyimide composite coatings with $10 \%$ (a), $20 \%$ (b), $30 \%$ (c) alumina.

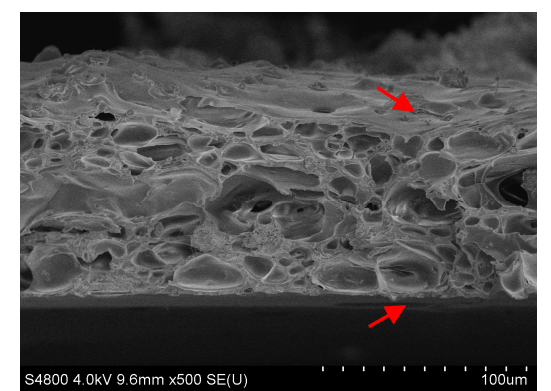

(a)

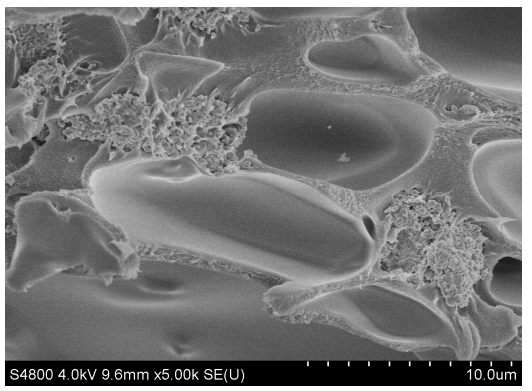

(d)

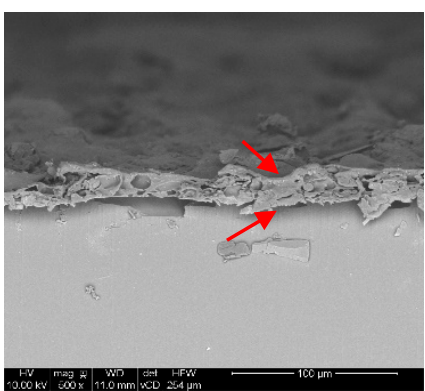

(b)

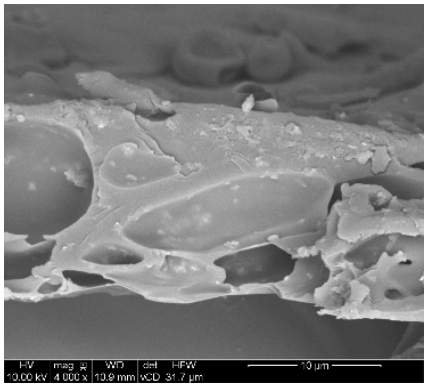

(e)

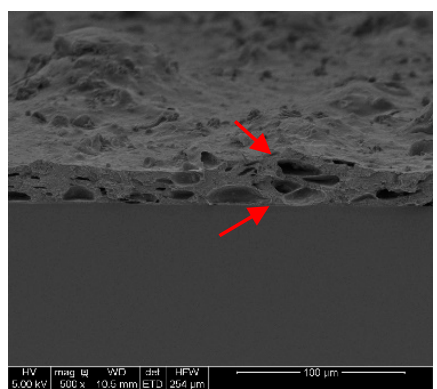

(c)

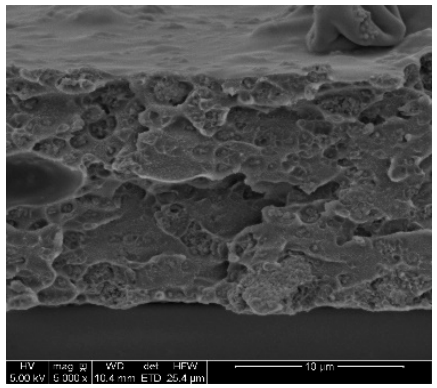

(f)

Figure 5. Cross-sectional structure of composite polyimide coatings with $10 \%(\mathbf{a}, \mathbf{d}), 20 \%(\mathbf{b}, \mathbf{e})$ and $30 \%$ $(\mathbf{c}, \mathbf{f})$ alumina. Arrows indicate top and bottom surface of coatings.

As polyimide is a polymer insulating material and is widely used to produce flexible circuit boards and motor slot insulating material, the dielectric coefficient of the polyimide $/ \mathrm{Al}_{2} \mathrm{O}_{3}$ composite coatings were examined and shown in Figure 6. It is seen that the dielectric constant of the composite coating is between 1.4 and 1.9, and the dielectric constant of the composite coating does not change much with the frequency for all coatings. However, the dielectric constant of the polyimide-alumina composite 
coating increases with the alumina content. Dielectric constant is closely related to the characteristics of the material itself and the density of polarized molecules in the material [16]. It is known that the dielectric constant of the pure polyimide film is generally between 3.0 and 3.4, and that of alumina is 8.6-10 $[17,18]$. With the increase in alumina amount, the number of polar groups and phase interface between the organic phase and the inorganic phase in the composite coating increases, which might lead to an increase in chemical capacity and the dielectric coefficient increases. Moreover, the density of polarized molecules in the material has great impact on the dielectric constant. The polyimide coating prepared by thermal spray has many holes, which greatly reduce the density of polarized molecules in the material, thereby effectively reducing its dielectric coefficient compared with pure polyimide films.

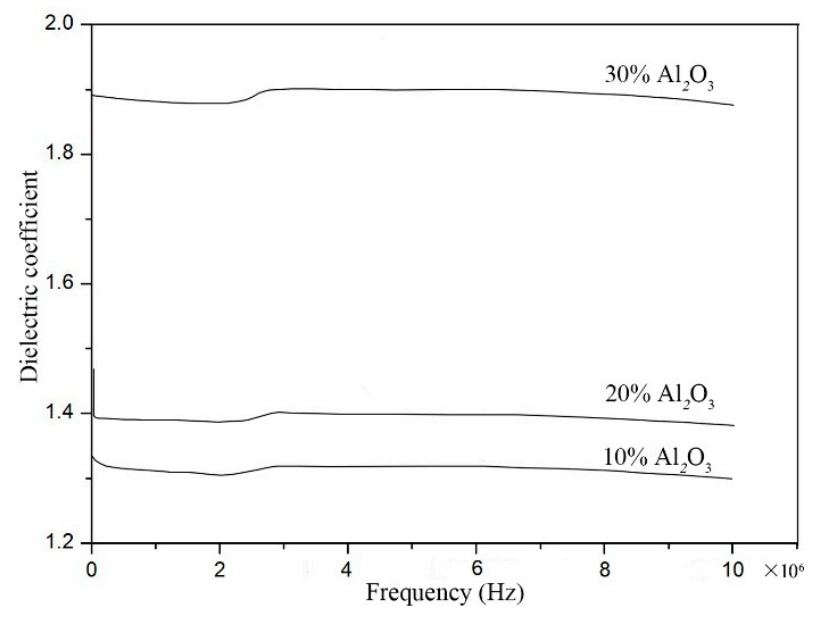

Figure 6. The dielectric coefficient polyimide/alumina composite coatings with different content of alumina.

\section{Conclusions}

A one-step fabrication of polyimide coatings using liquid flame spray was successfully established using a PAA precursor solution. Furthermore, the addition of alumina into the precursor solution resulted in facile incorporation into the coating at the same time, which leads to composite coatings with enhanced compactness and reduced porosity. The polyimide-alumina composite coatings exhibit excellent dielectric properties. The cost-effective large-scale fabrication technique for making the polymer-inorganic-based coatings sheds light on constructing electrical insulation materials and the fabrication of polymer-based composites for various applications.

Author Contributions: Data curation, J.Z. and Y.Z.; Formal analysis, J.Z., K.S. and Y.Z.; Investigation, J.Z., K.S., S.H., X.H. and W.C.; Writing-original draft preparation, J.Z.; Funding acquisition, W.L.; Project administration, W.L.; Supervision, W.L.; Methodology, Y.Z.; Writing—review and editing, Y.Z. All authors have read and agreed to the published version of the manuscript.

Funding: This research was funded by China Southern Power Grid Co., Ltd. Liuzhou Bureau of EHV Transmission Company, Grant No. CGYKJXM2017039.

Acknowledgments: The authors thank Hua Li, Botao Zhang and Yi Liu from Ningbo Institute of Materials Technology and Engineering, Chinese Academy of Sciences, for the SEM measurements.

Conflicts of Interest: The authors declare no conflict of interest regarding the publication of this article. 


\section{References}

1. Luo, L.; Zheng, Y.; Huang, J.; Li, K.; Wang, H.; Feng, Y.; Wang, X.; Liu, X. High-performance copoly(benzimidazole-benzoxazole-imide) fibers: Fabrication, structure, and properties. J. Appl. Polym. Sci. 2015, 132, 42001. [CrossRef]

2. Sun, H.; Wang, F.; Shi, Y.; Zhou, J.; Wang, Y.; Li, Z.Y.; Jin, Z.; Hong, Z. Insulation characteristics of polyimide as insulation material used in pancake tape coil structure for resistive-type SFCL. IEEE Trans. Appl. Supercond. 2015, 25, 1-4. [CrossRef]

3. Zhou, Y.; Chen, Y.; Wang, H.; Wong, C.P. Creation of a multilayer aluminum coating structure nanoparticle polyimide filler for electronic applications. Mater. Lett. 2014, 119, 64-67. [CrossRef]

4. Ansari, R.; Hassanzadeh-Aghdam, M.K.; Darvizeh, A. Coefficients of thermal expansion of carbon nanotube-reinforced polyimide nanocomposites: A micromechanical analysis. Proc. Inst. Mech. Eng. Part L J. Mater. 2016, 233, 169-179. [CrossRef]

5. Ning, W.; Wang, Z.; Liu, P.; Zhou, D.; Yang, S.; Wang, J.; Li, Q.; Fan, S.; Jiang, K. Multifunctional super-aligned carbon nanotube/polyimide composite film heaters and actuators. Carbon 2018, 139, 1136-1143. [CrossRef]

6. Jang, H.G.; Yang, B.; Khil, M.-S.; Kim, S.Y.; Kim, J. Comprehensive study of effects of filler length on mechanical, electrical, and thermal properties of multi-walled carbon nanotube/polyamide 6 composites. Compos. Part A Appl. Sci. Manuf. 2019, 125, 105542. [CrossRef]

7. Li, X.; Fang, X.; Zhang, P.; Yan, J.; Chen, Y.; Chen, X. Preparation and properties of reduced graphene oxide/polyimide composite films. High Perform. Polym. 2020, 32, 65-72. [CrossRef]

8. Jia, W.; Wang, J.; Ma, L.; Ren, S.; Yang, S. Mechanical properties and thermal stability of porous polyimide/hollow mesoporous silica nanoparticles composite films prepared by using polystyrene microspheres as the pore-forming template. J. Appl. Polym. Sci. 2020, 137, 48792. [CrossRef]

9. Pavlenko, V.I.; Bondarenko, G.G.; Cherkashina, N.I. Physicomechanical characteristics of composite based on polyimide matrix filled with tungsten oxide. Inorg. Mater. Appl. Res. 2020, 11, 304-311. [CrossRef]

10. Mohamed, M.G.; Kuo, S.W. Functional polyimide/polyhedral oligomeric silsesquioxane nanocomposites. Polymers 2019, 11, 26. [CrossRef] [PubMed]

11. Ma, L.; Wang, B.; Zhao, S.; Hao, C.; Guo, H.; Lei, Q. The fabrication and electrical properties of polyimide/boron nitride nanosheets composite films. J. Mater. Sci. Mater. Electron. 2019, 30, 20302-20310. [CrossRef]

12. Ji, J.; Deng, C.; Liu, X.; Qin, J. Fabrication of porous polyimide hollow microspheres through o/w/o multiple emulsion. Colloids Surf. A Physicochem. Eng. Asp. 2020, 591, 124537. [CrossRef]

13. Cherkashina, N.I.; Pavlenko, V.I.; Noskov, A.V. Radiation shielding properties of polyimide composite materials. Radiat. Phys. Chem. 2019, 159, 111-117. [CrossRef]

14. Tian, H.; Wang, C.; Guo, M.; Cui, Y.; Gao, J.; Tang, Z.; Liang, Y.; Song, C.; Wang, H.; Jin, G.; et al. Study on process and performance of thermal protective coating on polyimide resin matrix composite. Ceram. Int. 2020, 46, 12744-12758. [CrossRef]

15. Gouzman, I.; Grossman, E.; Verker, R.; Atar, N.; Bolker, A.; Eliaz, N. Advances in polyimide-based materials for space applications. Adv. Mater. 2019, 31, 1807738. [CrossRef] [PubMed]

16. Xu, R.; Ma, J.; Zhou, R.; Sun, H.; Xu, D.; Zeng, Z. Black phosphorus nanoflakes/polyimide composite films with excellent dielectric and mechanical properties. J. Mater. Sci. Mater. Electron. 2020, 31, 3303-3311. [CrossRef]

17. Yang, K.; Kang, Y.Y.; Ahn, H.J.; Kim, D.G.; Park, N.K.; Choi, S.Q.; Won, J.C.; Kim, Y.H. Porous boron nitride/polyimide composite films with high thermal diffusivity and low dielectric properties via high internal phase pickering emulsion method. J. Ind. Eng. Chem. 2020, 82, 173-179. [CrossRef]

18. He, S.; Hu, J.; Zhang, C.; Wang, J.; Chen, L.; Bian, X.; Lin, J.; Du, X. Performance improvement in nano-alumina filled silicone rubber composites by using vinyl tri-methoxysilane. Polym. Test. 2018, 67, 295-301. [CrossRef]

(C) 2020 by the authors. Licensee MDPI, Basel, Switzerland. This article is an open access article distributed under the terms and conditions of the Creative Commons Attribution (CC BY) license (http://creativecommons.org/licenses/by/4.0/). 